\title{
Evidence of mesogranulation from magnetograms of the Sun
}

\author{
I. Domínguez Cerdeña ${ }^{\star}$ \\ Universitäts-Sternwarte, Geismarlandstraße 11, 37083 Göttingen, Germany \\ Received 29 October 2003 / Accepted 14 November 2003

\begin{abstract}
This work confirms the existence of the mesogranulation as an intermediate scale of convection in the solar photosphere. The main argument presented is the correlation between horizontal flows obtained with two different methods (Local Correlation Tracking, and gradient of granular intensities), and the pattern observed in magnetograms above a fixed threshold of the magnetic signal. The data used consist of a time series (40 min) of high spatial resolution images of intensity and simultaneous magnetograms. The size of the scales found is $5^{\prime \prime}-10^{\prime \prime}(3.6-7.2 \mathrm{Mm})$, but some mesogranules close to supergranular
\end{abstract} \\ boundaries can be smaller, and appear to be stabilized by the network magnetic fields.
}

Key words. Sun: granulation - Sun: magnetic fields

\section{Introduction}

The study of convection in the Sun (and in stars in general) is one of the main subjects in solar physics. Many works have been inspired not only by its importance for the transport of energy but also due to its essential influence on the solar magnetism. Convection at the solar surface is characterized by the presence of cellular patterns with three different scales. The most obvious is the granulation, which is seen in intensity images and was observed for the first time by Herschel in 1801. The size of the granules is about $1 \mathrm{Mm}$, and their lifetime is about $8 \mathrm{~min}$. The next scale discovered was the supergranulation (or macrogranulation), which was observed by Leighton et al. (1962). This scale is visible as a pattern in horizontal velocities, so it can be observed with Dopplegrams, having a typical size of 20-30 Mm and lifetimes of the order of 20 hours. Finally, November et al. (1981) found the mesogranulation, a scale in between, with sizes of 3-10 Mm and lifetimes between 3 and 10 hours. It can be observed from Doppler measurements of vertical velocities, or from tracking of granules. Mesogranules seem to be advected to the boundaries of supergranules (Muller et al. 1992; Shine et al. 2000) disappearing on their way.

The origin of the three scales is sometimes related to the ionization of $\mathrm{H}, \mathrm{He}$ and $\mathrm{He}^{+}$. The depths in the solar interior where these three ionizations occur appear to coincide with the three scales in convection. However different origins have been suggested from simulations. For example Cattaneo et al. (2001) explain mesogranules as collective interactions between granules.

The relation between convection and magnetic field has been under study for many years. On the one hand, it is well known that the magnetic flux in the photosphere is

\footnotetext{
* e-mail: ita@uni-sw.gwdg.de
}

concentrated in the boundaries of the supergranules (Simon $\&$ Leighton 1964). The result of such a concentration is the chromospheric network, where magnetic field processes lead to an increased energy supply (Simon et al. 2001). On the other hand, granulation may be the origin of most of photospheric magnetic flux produced by a local dynamo (Cattaneo 1999). Granules may generate magnetic fields in the interior of supergranules, in the so called internetwork, which may harbour most of the unsigned magnetic flux on the solar surface (e.g. Sánchez Almeida et al. 2003, and references therein). The residuals from such fields can be transported to the boundaries of supergranules by the horizontal flows. Magnetoconvection simulations (Cattaneo 1999; Stein \& Nordlund 2002; Vögler \& Schüssler 2003) suggest that at the granular scale, the magnetic fields are concentrated in the boundaries of the cellular patterns (in intergranular lanes) also driven by the granular horizontal flows, and observational evidence has recently been presented (e.g Lin \& Rimmele 1999; Domínguez Cerdeña et al. 2003a; Khomenko et al. 2003). As this magnoto-convective concentration exists for these two scales, it is reasonable to adopt that it also occurs at mesogranular scales. Such a possibility has been predicted by numerical simulations (Cattaneo et al. 2001), and it has been observed very recently with twodimensional spectro-polarimetry (Domínguez Cerdeña et al. 2003b), IR spectro-polarimetry (Trujillo Bueno 2003), and from magnetograms (Sánchez Almeida 2003).

In recent years there has been a controversy about the reality of the existence of the mesogranules as an intermediate scale in addition to the granular and supergranular scales, due to the large range of scales and lifetimes deduced by different authors from different observations (see Rieutord et al. 2000).

This work employes for the first time two independent ways to detect mesogranulation: the horizontal flows, and the concentration of magnetic flux. The observations and 
pre-processing of the data are explained in Sect. 2. The deduction of the horizontal flows and the results are disscused in Sect. 3. The final conclusions are presented in Sect. 4.

\section{Observations and data processing}

The obervations are the same as those used by Stenflo \& Holzreuter (2003) and by Sánchez Almeida (2003). The data were obtained by G. Scharmer on February 9, 1996 using the SVST (Swedish Vacuum Solar Telescope) at the Observatorio del Roque de los Muchachos. They consist of a time series of $40 \mathrm{~m}$ duration of solar granulation images and of maps of circular polarization in the wing of the Fe I $15250.2 \AA$ line, which is sensitive to magnetic fields. The observations were taken from quiet Sun close to disc centre and include some network patches. The field of view (FOV) was $59^{\prime \prime} \times 81^{\prime \prime}$ with a pixel size of $0{ }^{\prime} 16$. The time series contains 44 images taken with a cadence of 56 s. Sánchez Almeida (2003) estimated the spatial resolution to be $0{ }^{\prime} 5$. He also established a calibration of the magnetograms provided as degree of circular polarization $V / I$.

The original data had to be prepared for the temporal analysis. Global shifts produced by seeing were removed from each snapshot. There is a rotation of the FOV during the series, due to the altitude-azimuth mount of the SVST. We can correct for both effects by computing local shifts at different points in the FOV using correlation on granulation. It is possible to obtain from these shifts a global shift and an angle of rotation.

There are two more effects to be removed, oscillations (p-modes) and the differential seeing, which cause a local change in intensity. For that purpose a subsonic filter is applied as proposed by Title et al. (1989), with a cutoff phase velocity of $5 \mathrm{~km} \mathrm{~s}^{-1}$. This value seems to be enough to clean most of these effects. This cutoff was already used by Hirzberger et al. (1997) for a detailed analysis of granulation.

This last step involves an edge filtering (apodization), as many operations are done in Fourier space. After the apodization the FOV is reduced to $320 \times 436$ pixels or $53^{\prime \prime} \times 72^{\prime \prime}$, and the time series consists now of 38 images (and magnetograms), with a 35 min duration.

\section{Results}

\subsection{Horizontal flows}

One of the traditional ways to detect the mesogranules is to compute their horizontal velocities using local correlation tracking (LCT, November \& Simon 1989). The FWHM of the Gaussian correlation for the LCT was fixed to 2'.5. Binary maps were produced from the resulting images, so the algorithm gives the movement of the granules. The mean velocity found is approximately $400 \mathrm{~m} \mathrm{~s}^{-1}$, with an rms of $360 \mathrm{~m} \mathrm{~s}^{-1}$. The vertical velocity can be obtained from the divergence of the horizontal velocity,

$u_{z}=h\left(\boldsymbol{\nabla} \cdot \boldsymbol{u}_{\boldsymbol{h}}\right)$

with $h$ the mass-flux scale height ( $h=150 \mathrm{~km}$, see November 1989). The vertical velocity is plotted in Fig. 1, it has an rms of $50 \mathrm{~m} \mathrm{~s}^{-1}$.

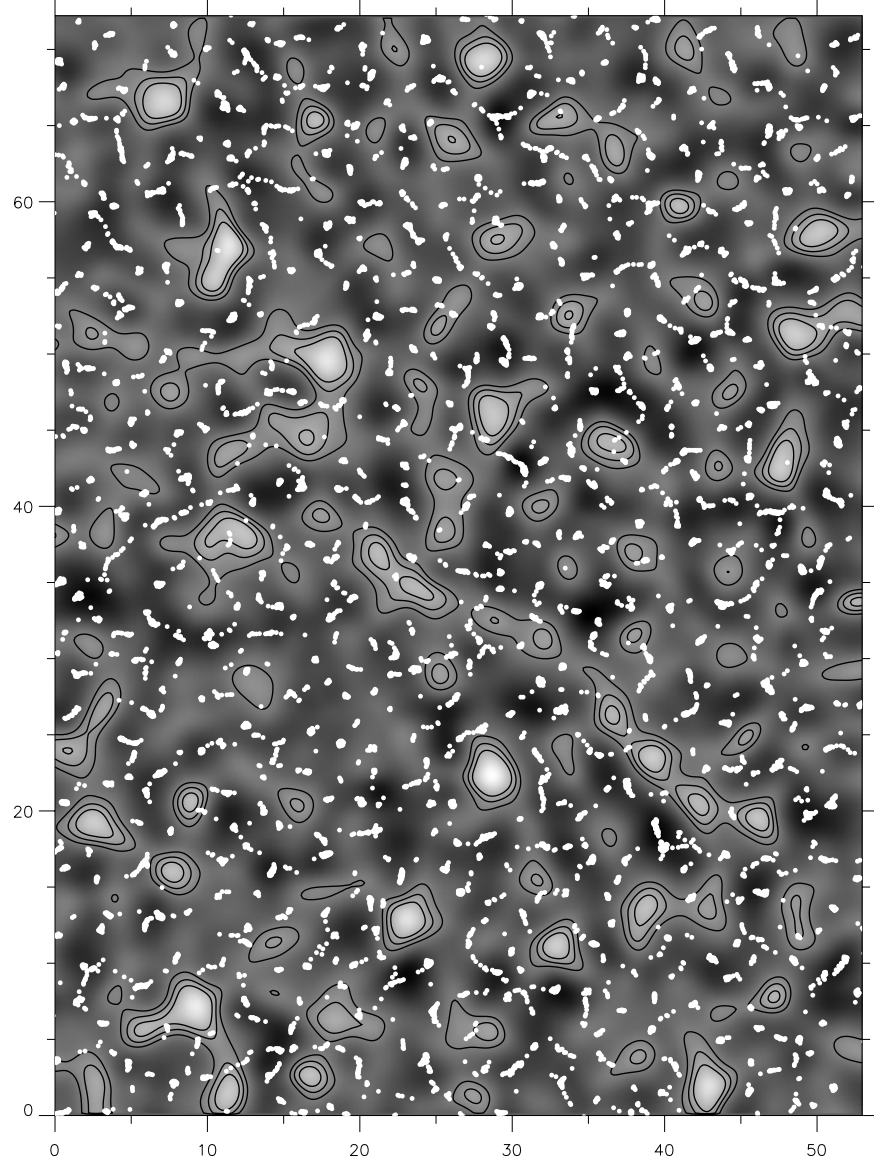

Fig. 1. Vertical velocity obtained from the divergence of the horizontal flow computed with LCT. Black contours delineate upward flows with velocities of 40,70 and $100 \mathrm{~m} \mathrm{~s}^{-1}$ showing the centres of clear mesogranules. White dots show the final position of the corks (cf. Sect. 3.1). The scales on the axes are in arcsec.

Another way to trace the mesogranulation is to extract the instantaneous horizontal velocity from the intensity images. Under the assumption of small vertical vorticity in the granulation, as in van Ballegooijen et al. (1998), the velocity can be aproximated by a potential flow field according to

$v=-\nabla \phi$.

The potential $\phi$ is supposed to be correlated with the intensity, so it is possible to obtain horizontal velocities for each individual image of the series. The gas flows from the centres of the granules to the intergranular lanes, which is the intuitive motion of the plasma. As the proportionality between temperature and intensity is uncertain it is necessary to scale the velocities. We assume that the rms of each velocity component is $1.3 \mathrm{~km} \mathrm{~s}^{-1}$, obtained from three-dimensional numerical simulations of granulation with the MURAM code (Vögler \& Schüssler 2003). (MURAM solves the MHD equations for a compressible and partially ionized plasma, taking into account radiative transfer. For the present purpose, a purely hydrodynamical solution is used.)

To assume a potential flow field is far from reality for the small details of the dynamics, however it should be useful to study the large-scale motion. An appropriate way to analyse the 


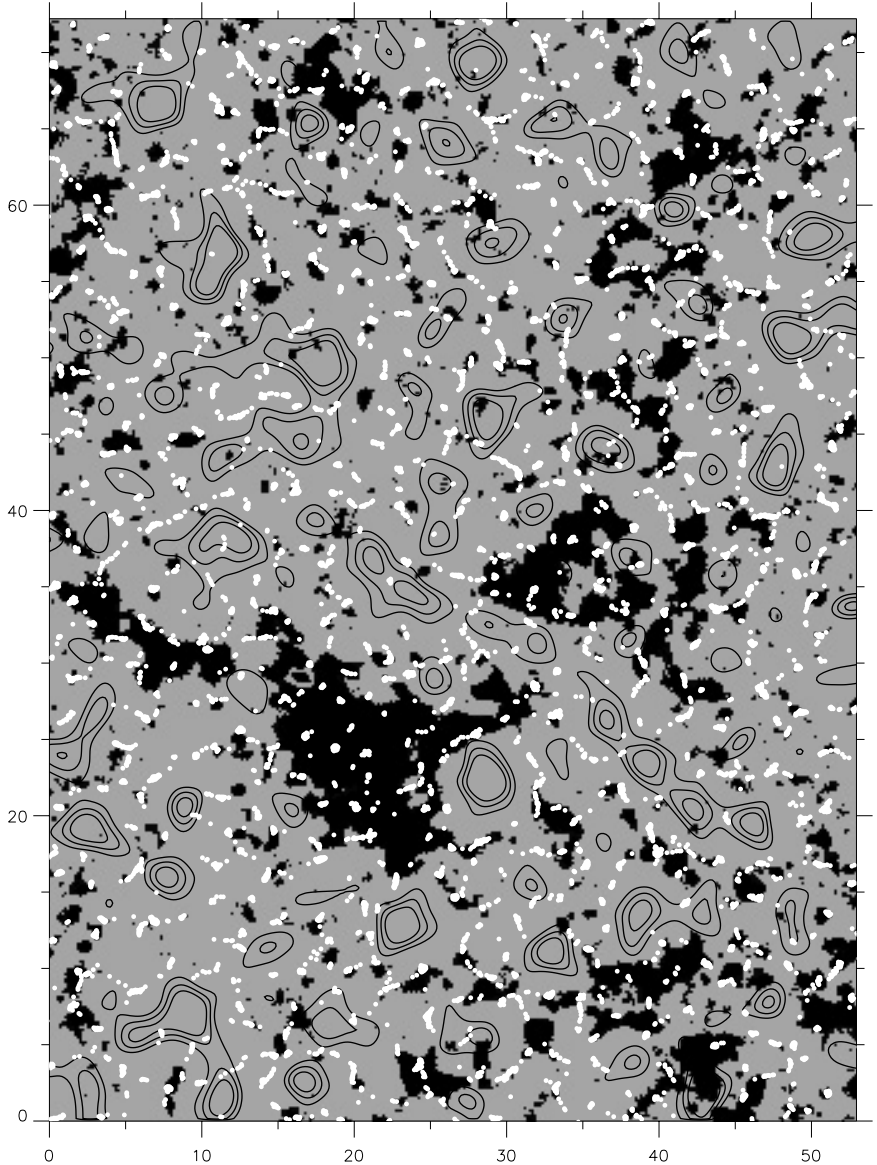

Fig. 2. Magnetogram as a binary map. Black represents absolute magnetic flux above $60 \mathrm{G}$, and grey below $60 \mathrm{G}$. Contours and dots are as in Fig. 1.

simulation is the use of corks, imaginary particles moving on the surface following the velocities calculated from Eq. (2). At the beginning, 8700 corks are homogenously distributed over the FOV. The final positon of the corks after $35 \mathrm{~min}$ of evolution is plotted in Fig. 1. The result of the two methods for computing horizontal flows is the expected mesogranular pattern, with sizes of $5^{\prime \prime}-10^{\prime \prime}$. The highest values of vertical upward velocity (black contours) are clearly coaligned with the big areas empty of corks resulting from the stable flow of mesogranules.

\subsection{Correlation with magnetic field}

Figure 2 shows a binary map of a magnetogram from the end of the time series (after $32 \mathrm{~m}$ of elapsed time). Pixels with absolute magnetic flux density above $60 \mathrm{G}$ are black and below $60 \mathrm{G}$ are grey. The network is easily identified as the biggest areas with magnetic field. The FOV covers one supergranule (top and left), and part of two others. High values of the divergence (contours) and corks are plotted as in Fig. 1. Magnetic flux is predominantly concentrated at the boundaries of the mesogranules identified by high values of the vertical velocity. In many parts of the FOV it is possible to see a cellular pattern in magnetic flux which is well correlated with the cells traced by corks. But one must not expect to see this everywhere. Some "cork cells" have only a few flux concentrations here and there, as it usually happens also with supergranules and network.

Contrary to some works which suggest that mesogranulation becomes weaker close to supergranular boundaries (Shine et al. 2000), it is possible to see many mesogranular flows in contact with the strong magnetic fields which form the network in this FOV. These places present an even more clear and stable cellular structure where, possibly, the convection at this scale is enhanced by magnetic fields (e.g. at positions [29", $\left.22^{\prime \prime}\right],\left[38^{\prime \prime}, 13^{\prime \prime}\right]$, and [37", 43"]). On the other hand some of the mesogranular cells close to the network have smaller sizes than mesogranules in the Internetwork. Such structures can appear in form of high divergences (e.g. [41" $\left.60^{\prime \prime}\right],\left[18^{\prime \prime}, 66^{\prime \prime}\right]$ ) or only as regions free of corks (e.g. [39", 52"] $\left.\left[46^{\prime \prime}, 4^{\prime \prime}\right]\right)$.

\section{Conclusions}

The use of a time series of simultaneous quiet Sun intensity images and magnetograms provides a new argument on the existence of a scale of convection in-between granulation and supergranulation. The map of divergence (vertical velocity in Fig. 1) obtained from the horizontal flows computed with LCT gives an idea of the position of mesogranules. These positions are in agreement with the cork evolution using velocities derived from intensity gradients of individual images. The main result is that the magnetograms show how the boundaries of such structures are enriched with magnetic flux (Fig. 2) which seems to be carried by the horizontal motions of the plasma.

The size of the mesogranulation covers a range between $5^{\prime \prime}$ and $10^{\prime \prime}$ (never more), and the use of corks and especially of magnetograms avoid the dependence of the size with the smoothing window used in the LCT technique. The presence of clearly outlined and stable mesogranules interacting with strong magnetic fields of the network has been observed, contradicting the idea of the absence of mesoscales at the boundaries of supergranules. Moreover some of the cells interacting with the network are smaller in size than the ones observed in the center of supergranules (as was already observed by Berger et al. 1998). All this suggests that the magnetic field plays a role in the evolution and possibly in the formation of the mesogranules.

Magnetograms obtained with high resolution and considerable magnetic sensitivity have become a new tool in the study of mesogranulation. This not only gives a new probe to confirm the existence of the mesogranulation and to investigate its structure and evolution, but also can be used to complete the puzzle of the relation between magnetic field and convection.

Acknowledgements. The magnetograms were obtained by G. Scharmer with the SVST operated by the Royal Swedish Academy of Sciences in the Spanish Observatorio del Roque de los Muchachos. Thanks are due to F. Kneer, J. A. Bonet, J. Sánchez Almeida, J. Trujillo Bueno and R. Muller for useful discussion on this work and for comments on the manuscript. IDC acknowledges support by the Deutsche Forschungsgemeinschaft (DFG) through grant 418 SPA-112/14/01. 


\section{References}

Berger, T. E., Loefdahl, M. G., Shine, R. S., \& Title, A. M. 1998, ApJ, 495, 973

Cattaneo, F. 1999, ApJ, 515, L39

Cattaneo, F., Lenz, D., \& Weiss, N. 2001, ApJ, 563, L91

Domínguez Cerdeña, I., Kneer, F., \& Sánchez Almeida, J. 2003a, ApJ, 582, L55

Domínguez Cerdeña, I., Sánchez Almeida, J., \& Kneer, F. 2003b, A\&A, 407, 741

Hirzberger, J., Vazquez, M., Bonet, J. A., Hanslmeier, A., \& Sobotka, M. 1997, ApJ, 480, 406

Khomenko, E. V., Collados, M., Solanki, S. K., Lagg, A., \& Trujillo Bueno, J. 2003, A\&A, 408, 1115

Leighton, R., Noyes, R. W., \& Simon, G. W. 1962, ApJ, 135, 474

Lin, H., \& Rimmele, T. 1999, ApJ, 514, 448

Muller, R., Auffret, H., Roudier, T., et al. 1992, Nature, 356, 322

November, L. J. 1989, ApJ, 344, 494

November, L. J., \& Simon, G. W. 1988, ApJ, 333, 427

November, L. J., Toomre, J., Gebbie, K. B., \& Simon, G. W. 1981, ApJ, 245, L123
Rieutord, M., Roudier, T., Malherbe, J. M., \& Rincon, F. 2000, A\&A, 357,1063

Sánchez Almeida, J. 2003, A\&A, 411, 615

Sánchez Almeida, J., Emonet, T., \& Cattaneo, F. 2003, ApJ, 585, 536

Shine, R. A., Simon, G. W., \& Hurlburt, N. E. 2000, Sol. Phys., 193, 313

Simon, G. W., \& Leighton, R. B. 1964, ApJ, 140, 1120

Simon, G. W., Title, A. M., \& Weiss, N. O. 2001, ApJ, 561, 427

Stein, R. F., \& Nordlund, A. 2002, ESA SP-505: SOLMAG 2002. Proceedings of the Magnetic Coupling of the Solar Atmosphere Euroconference, 83

Stenflo, J. O., \& Holzreuter, R. 2003, Astron. Nachr., 324, 397

Title, A. M., Tarbell, T. D., Topka, K. P., et al. 1989, ApJ, 336, 475

Trujillo Bueno, J. 2003, in Modeling of Stellar Atmospheres, ed. N. E. Piskunov, W. W. Weis, \& D. F. Gray, IAU Sympos. 210, ASP Conf. Ser., in press

van Ballegooijen, A. A., Nisenson, P., Noyes, R. W., et al. 1998, ApJ, 509,435

Vögler, A., \& Schüssler, M. 2003, Astron. Nachr., 324, 399 